\title{
Spin Glasses: Still Complex After All These Years?
}

\author{
D.L. Stein \\ Departments of Physics and Mathematics, University of Arizona, Tucson, AZ 85721 \\ USA
}

\begin{abstract}
Spin glasses are magnetic systems exhibiting both quenched disorder and frustration, and have often been cited as examples of 'complex systems.' In this talk I review some of the basic notions of spin glass physics, and discuss how some of our recent progress in understanding their properties might lead to new viewpoints of how they manifest 'complexity'.
\end{abstract}

\section{Introduction}

This talk will probably be a change of pace for most of you; at least the topic is mostly orthogonal to those covered by the other talks. In particular, I'll be discussing a classical statistical mechanical problem. The origins of the interactions that define the spin glass are of course quantum mechanical; and quantum phenomena in many spin glass systems have become an active area of study over the past decade. Nevertheless, many of the important phenomena observed down to very low temperatures in a wide variety of spin glasses can be explained using classical statistical mechanics - or, more truthfully, could be explained if we could figure out how to treat the enormous complications arising from the quenched randomness inherent in these systems. Because of these complications, the most basic questions remain open, and the spin glass has often been touted as a model example of a complex system.

In the absence of a universally agreed definition of 'complex system', it is as difficult to argue with that claim as it is to justify it. Maybe spin glasses - as well as other systems discussed at this meeting - are merely 'complicated systems'. What I'll try to do in this talk is to convey some of the flavor of spin glass physics, and to show why its understanding requires the introduction of some new concepts and tools into statistical mechanics. You can then judge for yourself whether the classical spin glass fits your own understanding of 'complexity'.

The talk will be divided into four parts:

- What is a spin glass?

- Why are they interesting to physicists?

- What is the current level of our understanding?

- What - if anything - do they have to do with complexity? 


\section{Brief Review of Spin Glasses}

Spin glasses are systems with localized electronic magnetic moments whose interactions are characterized by quenched randomness: a given pair of localized moments ('spins' for short) have a roughly equal a priori probability of having a ferromagnetic or an antiferromagnetic interaction. The prototype material is a dilute magnetic alloy, with a small amount of magnetic impurity randomly substituted into the lattice of a nonmagnetic metallic host; for example, $C u \mathrm{Mn}$ or $A u \mathrm{Fe}$. However, insulators such as $\mathrm{Eu}_{x} \mathrm{Sr}_{1-x} \mathrm{~S}$, with $x$ roughly between .1 and .5, also display spin glass behavior. The underlying physics governing the random interactions differs for different classes of materials; for the dilute magneitc alloys, it arises from the conduction electron-mediated RKKY interactions between the localized moments.

Early experiments by Cannella and Mydosh [1] indicated that a phase transition occurred in $A u$ Fe alloys: the low-field ac magnetic susceptibility exhibited a cusp at a frequency-dependent temperature $T_{f}$. Similar behavior has since been seen in other spin glasses, and has become a signature feature of spin glass behavior. At the same time, specific heat curves show no singularities, but instead a smoothly rounded maximum at a temperature slightly above $T_{f}$ (for a review, see [2]). Whether there exists a true thermodynamic phase transition to a low-temperature spin glass phase remains an open question.

Neutron magnetic scattering data and other probes of magnetic structure indicate that at low temperatures, the spins are frozen - at least on experimental timescales - in random orientations. Hence the name spin glass: the magnetic disorder is reminiscent of the translational disorder in the atomic arrangement of an ordinary glass.

\section{Why Should We Care?}

So why should we care?

- Because it's there. From the perspective of condensed matter physics, any new class of condensed matter systems is worth understanding. Of course, some classes of systems are more interesting than others. By more interesting, I mean that they may have great importance for technological application, and/or they give rise to powerful new ideas, and perhaps new physical or mathematical tools. Often these ideas and tools are applicable to different kinds of condensed matter systems, and perhaps to problems outside of condensed matter physics altogether. A well-known example is the broken gauge symmetry of superconductors providing a 'mass' to the photon, which was influential in the uncovering of the Higgs mechanism in particle physics.

Spin glasses are likely to belong to such a class of systems; indeed, as we'll describe below, they have already proved a fertile ground for uncovering new ideas and techniques with potentially wide applicability. But returning to the problem of spin glasses proper: unlike ordinary glasses, which must be cooled sufficiently rapidly to avoid the crystalline phase, the spin glass has no competing 
ordered phase. So if a thermodynamic phase transition does exist, then the low temperature phase would truly be an equilibrium condensed disordered phase a new state of matter.

- Statistical Mechanics of Disordered Systems. Homogeneous systems, such as crystals, uniform ferromagnets, and superfluids, display spatial symmetries that greatly simplify their physical and mathematical analyses. The absence of such symmetries enormously complicates the understanding of disordered systems like spin glasses. This may lead to new types of broken symmetries, a breakdown of the thermodynamic limit for certain quantities, the emergence of new phenomena such as chaotic temperature dependence, the need for creation of new thermodynamic tools, and other unanticipated features to be described below. While it may not be necessary to completely revamp statistical mechanics in order to understand disordered systems, as has sometimes been suggested, it is at least necessary to carefully rethink some deeply held assumptions.

- Applications to Other Areas. Concepts that arose in the study of spin glasses have led to applications in areas as diverse as computer science 3456], neural networks [78, prebiotic evolution [91011], protein conformational dynamics [12], protein folding [13, and a variety of others. We will not have time to discuss these applications here, but extensive treatments can be found in 141516 .

\section{Spin Glass Theory}

The modern theory of spin glasses began with the work of Edwards and Anderson (EA) 17, who proposed that the essential physics of spin glasses lay not in the details of their microscopic interactions but rather in the competition between quenched ferromagnetic and antiferromagnetic interactions. It should therefore be sufficient to study the Hamiltonian

$$
\mathcal{H}_{\mathcal{J}}=-\sum_{<x, y>} J_{x y} \sigma_{x} \sigma_{y}-h \sum_{x} \sigma_{x},
$$

where $x$ is a site in a $d$-dimensional cubic lattice, $\sigma_{x}= \pm 1$ is the Ising spin at site $x, h$ is an external magnetic field, and the first sum is over nearest neighbor sites only. To keep things simple, we take $h=0$ and the spin couplings $J_{x y}$ to be independent Gaussian random variables whose common distribution has mean zero and variance one. With these simplifications, the EA Hamiltonian (10) has global spin inversion symmetry. We denote by $\mathcal{J}$ a particular realization of the couplings, corresponding physically to a specific spin glass sample.

\subsection{Frustration}

The Hamiltonian (11) exhibits frustration: no spin configuration can simultaneously satisfy all couplings. If a closed circuit $\mathcal{C}$ in the edge lattice satisfies the property

$$
\prod_{<x, y>\in \mathcal{C}} J_{x y}<0
$$




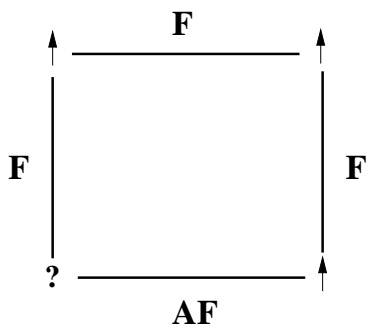

Fig. 1. A simple frustrated contour in a $2 D$ lattice. Bonds marked "F" correspond to ferromagnetic couplings $\left(J_{x y}>0\right)$ and "AF" corresponds to an antiferromagnetic coupling $\left(J_{x y}<0\right)$. One possible arrangement of spins at the corner sites is shown.

then the spins along it cannot all be simultaneously satisfied 18 (Fig. 1).

Anderson [19] suggested a different formulation, namely that frustration manifests itself as free energy fluctuations scaling as the square root of the surface area of a typical sample. Either way, the spin glass is characterized by both quenched disorder and frustration. Their joint presence indicates the possibility that spin glasses might possess multiple pure thermodynamic states unrelated by any simple symmetry transformation. We will return to this question later.

\subsection{Mean Field Theory}

Within months of appearance of the EA model, an infinite-ranged version was proposed by Sherrington and Kirkpatrick (SK) 20]. For a system of $N$ Ising spins, and in zero external field, their Hamiltonian is

$$
\mathcal{H}_{\mathcal{J}, N}=-\frac{1}{\sqrt{N}} \sum_{1 \leq i<j \leq N} J_{i j} \sigma_{i} \sigma_{j}
$$

where the independent, identically distributed couplings $J_{i j}$ are again chosen from a Gaussian distribution with zero mean and variance one; the $1 / \sqrt{N}$ rescaling ensures a sensible thermodynamic limit for free energy per spin and other thermodynamic quantities.

SK showed that their model had an equilibrium phase transition at $T_{c}=1$. While the static susceptibility had a cusp there, so did the specific heat. This wasn't necessarily surprising given that infinite-ranged models aren't expected to correctly describe the behavior of low-dimensional systems at the critical point. More troubling was SK's observation that the low-temperature phase had an instability: in particular, the entropy became negative at very low temperature.

A mean field theory, employing the Onsager reaction field term, was proposed two years later by Thouless, Anderson, and Palmer [21. Their approach indicated that there might be many low-temperature solutions, possibly corresponding to different spin glass 'phases'. (As a point of nomenclature, one should probably reserve use of the term 'mean field theory' for the TAP model. Nevertheless, to save space and time, I will follow general practice and use the term 
to refer to the infinite-ranged SK model also.) Other important early papers include the work of deAlmeida and Thouless 22, who considered the stability of the SK solution in the $h-T$ plane, and the dynamical work of Sompolinsky and Zippelius 232425.

We will not have time to discuss these papers here, and will focus instead on what is believed today to be the correct solution for the low-temperature phase of the SK model. This solution, due to Parisi [26, employed a novel ansatz and required several more years before a physical interpretation could be worked out 272829 . The picture that finally arose was that of a system with an extraordinary new kind of symmetry breaking, known today as 'replica symmetry breaking', or RSB, after the mathematical procedures used to derive it. The essential idea is that the low-temperature phase consists not of a single spin-reversed pair of states, but rather of "infinitely many pure thermodynamic states" 27, not related by any simple symmetry transformations. In the next section, we describe the qualitative features of the Parisi solution in greater detail.

\subsection{Broken Replica Symmetry}

It had been pointed out by EA that a correct description of the spin glass phase needs to reflect the lack of orientational spin order with the spin 'frozenness', or long-range order in time. Denoting by $\Lambda_{L}$ a cube of side $L$ centered at the origin, and $\langle\cdot\rangle$ a thermal average, the magnetization per spin in a pure phase

$$
M=\lim _{L \rightarrow \infty} \frac{1}{\left|\Lambda_{L}\right|} \sum_{x \in \Lambda_{L}}\left\langle\sigma_{x}\right\rangle
$$

should vanish (for a.e. $\mathcal{J}$ ), while

$$
q_{E A}=\lim _{L \rightarrow \infty} \frac{1}{\left|\Lambda_{L}\right|} \sum_{x \in \Lambda_{L}}\left\langle\sigma_{x}\right\rangle^{2}
$$

should not.

The quantity $q_{E A}$ measures the breaking of time-reversal symmetry, and is now known as the 'EA order parameter', but by itself is not sufficient to describe the broken symmetry of the SK spin glass phase. The correct order parameter needs to describe the structure and relationships among the infinitely many states present at low temperature. (We ignore here the problems inherent in defining 'pure state' for the SK model; for more discussion on this, see 30 3132.) To do this, we consider the overlap between two states $\alpha$ and $\beta$, at fixed $\mathcal{J}$ and $T$ :

$$
q_{\alpha \beta} \approx \frac{1}{N} \sum_{i=1}^{N}\left\langle\sigma_{i}\right\rangle_{\alpha}\left\langle\sigma_{i}\right\rangle_{\beta},
$$

where $\langle\cdot\rangle_{\alpha}$ is a thermal average in pure state $\alpha$.

Given the infinity of states, quantities referring to individual pure states are of little use, even if such things could be defined. What is really of interest is 
the distribution of overlaps. Consider choosing two pure states randomly and independently from the Gibbs distribution at fixed $N$; this will be a mixture over many pure states $\alpha$, with varying weights $W_{\mathcal{J}}^{\alpha}$ (dependence on $N$ and $T$ is suppressed for ease of notation). Let $P_{\mathcal{J}}(q) d q$ be the probability that the overlap of the two states lies between $q$ and $q+d q . P_{\mathcal{J}}(q)$ is commonly referred to as the Parisi overlap distribution. It is equal to

$$
P_{\mathcal{J}}(q)=\sum_{\alpha} \sum_{\beta} W_{\mathcal{J}}^{\alpha} W_{\mathcal{J}}^{\beta} \delta\left(q-q_{\alpha \beta}\right) .
$$

If there is a single pure state, such as the paramagnet at $T>T_{c}$, then $P_{\mathcal{J}}(q)$ is simply a $\delta$-function at $q=0$. For ferromagnets with free or periodic boundary conditions, there are only two pure states, namely the uniform positive and negative magnetization states, each appearing in any finite-volume Gibbs state with weight $1 / 2$. The overlap distribution function is now a pair of $\delta$-functions, each with weight $1 / 2$, located at $\pm M^{2}(T)$.

What about in the SK model? According to the Parisi solution, for fixed $\mathcal{J}$ and (large) $N$, it has the form qualitatively sketched in Fig. 2 The nontrivial nature of the overlap structure reflects the presence of many states (although only a handful have weights of $O(1)$ ) that are not related to each other by a simple symmetry transformation.

Even more interesting is the non-self-averaging of the overlap distribution function. Suppose a new coupling realization $\mathcal{J}^{\prime}$ is considered. Now, for any large $N$, the overlaps (except for the two at $\pm q_{E A}$, which are present for almost every $\mathcal{J}$ ) will appear at different values of $q$, and the set of corresponding weights will also differ. This is true no matter how large $N$ becomes.

Let $P_{N}(q)=\overline{P_{\mathcal{J}, N}(q)}$, where $\overline{[\cdot]}$ denotes an average over coupling realizations, and $P(q)=\lim _{N \rightarrow \infty} P_{N}(q)$. The resulting distribution $P(q)$ will be supported on all values of $q$ in the interval [ $\left.-q_{E A}, q_{E A}\right]$; a sketch is shown in Fig. 3]

Together $P_{\mathcal{J}}(q)$ and $P(q)$ can be thought of as describing the broken symmetry of the SK model below $T_{c}$. There is another famous feature, namely the ultrametricity of the overlaps. Instead of considering pairs of states, one now considers triples. The Parisi solution predicts that, for almost every fixed $\mathcal{J}$, the distances $d_{\alpha \beta}=q_{E A}-q_{\alpha \beta}$ among the three pairs will form the sides of an equilateral or an acute isosceles triangle. These strong correlations among the overlaps correspond to a tree-like, or hierarchical, structure of the states.

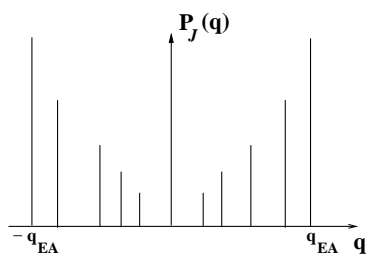

Fig. 2. Sketch of the overlap distribution function $P_{\mathcal{J}}(q)$ for the SK model below $T_{c}$. 


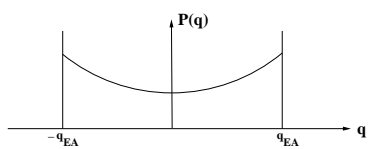

Fig. 3. Sketch of the averaged overlap distribution function $P(q)$ for the SK model below $T_{c}$. The spikes at $\pm q_{E A}$ are $\delta$-functions.

So the infinite-ranged spin glass has a strikingly novel type of broken symmetry, much different from anything observed in homogeneous systems. Because mean-field theory has usually provided a reliable description of the lowtemperature properties of finite-dimensional models, it was perhaps natural to expect that the RSB mean-field picture should similarly describe the nature of ordering in the EA and other short-ranged spin glass models. We now turn to these and related issues.

\subsection{Some open questions}

We saw from the preceding discussion that, already in mean field theory, spin glasses possess unusual and exotic properties. But what has been presented so far is only a piece of the story. Laboratory spin glasses display an array of puzzling thermodynamic and dynamical behavior. We confine ourselves here to a discussion of the nature of broken symmetry and ordering of the low-temperature spin glass phase - if there is one. At the same time, we emphasize that this represents only a part of the overall picture.

In this section we list some open - and fundamental - questions regarding the nature of broken symmetry in the short-ranged spin glass.

- Is there a phase transition to a low-temperature spin glass phase?

We have already seen that the answer is 'yes' for the infinite-ranged model. For the EA and other short-ranged models, the answer is not definitively known. There is some analytical 3334] and numerical [2 353637] work that supports existence of a phase transition in three - and even more likely, four - dimensional Ising spin glasses. But the issue remains unsettled [38. It is usually assumed (though it doesn't necessarily follow) that a low-temperature phase will be nonunique and display broken spin-flip symmetry - that is, a nonzero $q_{E A}$.

If there is no broken-spin-flip symmetric spin glass phase in any finite dimension, then the study of spin glasses becomes one of dynamics. In what follows we will simply assume that such a phase does exist, above some lower critical dimension. Then an important question is:

- Are there infinitely many pure state pairs below $T_{c}$ in the EA spin glass?

If RSB correctly describes the low- $T$ phase, then the answer is yes. However, a competing picture 39404142 434445 that arose in the early to mid-80's, based on domain-wall renormalization group ideas, leads to a very different picture of the low- $T$ phase. This approach, known as droplet/scaling, leads to the 
conclusion that there is only a single pair of spin-flip-reversed pure states at low temperature in any finite dimension.

Which of these alternatives is the case is not known in any dimension greater than one (where of course there is only a single pair). In two dimension, it is believed that $T_{c}=0$, so the issue becomes one of the number of ground state pairs only. Recent numerical experiments [6 4748] support the possibility of only a single pair of ground states. Recent rigorous work 4950] also supports the notion that only a single pair of ground states occurs in two dimensions. In three dimensions numerical simulations give conflicting results [5152].

But let's suppose that there are infinitely many ground and/or pure states above some lower critical dimension. Even if that's the case, it does not follow that the mean-field picture of replica symmetry breaking holds in finite dimensions, because that picture requires a very intricate pattern of relationships among all of the states. So we come to our third question:

- If there do exist infinitely many equilibrium states in some finite dimensions, is their organization similar to that of the Parisi solution of the SK model?

Here the question has largely been answered, due to a series of rigorous and heuristic results.

\subsection{Constraints on the Ordering of Short-Ranged Spin Glasses}

In a series of papers 305354555657, Newman and Stein have shown that the complex structure of replica symmetry breaking cannot be supported by finite-dimensional spin glasses in any dimension and at any temperature. The arguments will not be presented here, but instead we will focus on alternative scenarios that remain viable.

One possibility is that $T_{c}=0$ in any finite dimension, and all of the interesting features of spin glasses arise from dynamical processes. It could also happen that $T_{c}>0$, but there remains a unique (non-paramagnetic) Gibbs state below $T_{c}$. While either of these possibilities may end up being the case, it is the feeling of most workers in the field that there is a low-temperature phase with broken spin-flip symmetry.

If this is so, then 5354555657 leave open two main possibilities. (I emphasize here that these are not the only remaining logical possibilities, but rather the most plausible ones.) The first is a two-state picture like droplet/scaling, described in Sect.4.4 What about many-state pictures? There is one that would be consistent with the rigorous results of [535457, called the chaotic pairs picture 54565758 .

Consider a cube $\Lambda_{L}$ with $L$ large, and with periodic boundary conditions. In the chaotic pairs picture, the resulting finite-volume Gibbs state for any $0<$ $T<T_{c}$ would consist of a single pair of spin-flip-related pure states, just as in the droplet/scaling picture. But in the latter picture the same pair of pure states appears in every large volume, while in chaotic pairs the pure state pair appearing in $\Lambda_{L}$ will vary chaotically with $L$. 
So chaotic pairs resembles the droplet/scaling picture in any single finite volume, but differs over a collection of volumes and hence has a different thermodynamic structure. It is a many-state picture, but unlike the mean-field picture, it has a trivial overlap function. In any finite volume, $P_{\mathcal{J}}(q)$ would be a pair of $\delta$-functions at $\pm q_{E A}$, just as in the droplet/scaling picture. If one instead constructed the overlap function of the infinite-volume pure states, then it would most likely be a single $\delta$-function at the origin, for almost every $\mathcal{J}$. So, even though there are infinitely many states in this picture, there is no nontrivial replica symmetry breaking, no non-self-averaging, and no ultrametricity.

\section{$5 \quad$ Are Spin Glasses Complex Systems?}

The preceding overview enables us to return to the issue posed in the title of the talk. As I promised early on, I won't attempt to define 'complexity' or 'complex system', but will instead review some of the salient properties of spin glasses and leave it to you to decide whether they fit into your conception of a complex system. Whatever your answer, perhaps a more important characterization is whether you find them to be interesting and possibly relevant to problems that you work on. (And if the answer to all these is no, I hope that the talk at least was a pleasant diversion!)

I should begin by emphasizing that I did not cover, or even mention, many of the features of spin glasses that years ago helped to earn them the title of 'complex system'. These include some of the following: their property of displaying many metastable states, that is, states stable to flips of finite numbers of spins; their possessing a 'rugged energy landscape' (more or less equivalent to the preceding property, but sometimes also used to denote the presence of many pure or ground states); and their anomalous dynamical behaviors - slow relaxation, irreversibility, memory effects, hysteresis, and aging. I did briefly touch on their connections to problems in computer science, biology, and other areas, and of course that's important also.

But I'd like to emphasize here some of their more newly discovered properties that perhaps have not received as much attention. In what follows, I list several features - some recently uncovered, some not - in which spin glasses display unexpected behavior ('unexpected' meaning 'not familiar from our experience with the statistical mechanics of homogeneous systems'). A similar discussion appears in 31, and much of it represents joint work with Chuck Newman.

The appearance of broken replica symmetry in the infinite-ranged spin glass alone might merit the 'complexity' label, especially given its hierarchical structure in state space. A hierarchical organization of components has often been used to explain how a complex organization can be built out of simple components [59. However, it was noted above that this type of broken symmetry is absent in short-ranged spin glass models.

But this in itself is an interesting piece of news, because mean field theory has almost always served as an invaluable guide to the low-temperature behavior of statistical mechanical systems, in particular the nature of the order parameter 
and broken symmetry. The failure of mean field theory to provide a correct description of the low-temperature phase in any finite dimension indicates that the $d \rightarrow \infty$ limit of the EA model is singular. This possibility was broached by Fisher and Huse 44, and our work confirms their conjecture.

The failure of mean-field theory to describe the broken symmetry of shortranged models is perhaps just as interesting — and, if you're so inclined, just as good a candidate for the label 'complex' — as the exotic features of the Parisi solution. But why does mean field theory fail for realistic spin glasses? This is discussed at length in 3132, and I'll refer the interested reader to those papers. For those who are moderately interested, but not enough to begin reading yet another paper on this subject, I'll just note here that one important reason lies in the combination of the physical couplings scaling to zero in the SK model, along with their statistical independence. This combination ensures that something like the following must happen: suppose that some fixed $N_{1}$ corresponds to a particular 'pure state' structure (roughly speaking). As $N$ continues to increase, it will eventually reach an $N_{2} \gg N_{1}$ in which the earlier pure state structure, corresponding to $N_{1}$, is completely washed out. Any fixed, finite set of couplings will eventually have no effect on the spin glass state for $N$ large enough. In contrast, short-ranged models do not share this peculiar feature, or at least it should be considerably weaker there. For these models, the couplings outside of a particular fixed, finite region can act at most on its boundaries.

A second important feature is the possible nonexistence of a 'straightforward' thermodynamic limit for Gibbs states. By 'straightforward' I mean that a sequence of finite-volume Gibbs states, generated along an infinite sequence of volumes with boundary conditions chosen independently of the couplings, may not yield a limiting thermodynamic state. This should occur whenever there are many pure state pairs [58, although I haven't had time to discuss it here. But it's a reflection of the lack of any spatial symmetries that allow one to choose simple boundary conditions, like free or periodic, or an external symmetry-breaking field, that can lead to the existence of such a limit. (Two caveats: first, one can always choose subsequences that $d o$ lead to limiting thermodynamic states, but they would presumably have to be chosen in a coupling-dependent way, and at present there are no known ways of doing so. Second, this discussion is confined to Gibbs states, or equivalently correlation functions, which depend sensitively on the local details of the coupling realization. Global quantities, such as the free energy per spin, do have a limit for a given coupling-independent sequence of boundary conditions, for a.e. $\mathcal{J}$.)

This has an interesting consequence, related to our usual expectations for the behavior of large condensed matter systems. Statistical mechanical calculations typically rely on the assumption that the thermodynamic limit reveals the bulk properties of macroscopic systems. And in fact it almost certainly does, for disordered systems as well as homogeneous. But if, in the former case, there exist many competing pure states, then the connection between large (but finite) and infinite systems may be far more subtle than in homogeneous systems, where a straightforward extrapolation is generally sufficient. In order to connect the 
thermodynamic behavior of disordered systems with that of large but finite volumes, we have found it extremely useful to utilize a new thermodynamic tool which we call the metastate 5455565760 ,

Finally, it is possible that spin glasses display chaotic temperature dependence 41 45], in which correlation functions on sufficiently large lengthscales change in an unpredictable (though deterministic) fashion under infinitesimal temperature changes.

So the study of spin glasses has provided us with a host of new phenomena and tools: analytical tools such as replica symmetry breaking for infinite-ranged systems, and metastates for both short-ranged and infinite-ranged systems; numerical tools such as simulated annealing; and others. The infinite-ranged spin glass displays a beautiful new type of order, and the short-ranged spin glass seems to break many of the old rules and assumptions. Perhaps most surprisingly, analytical - and even rigorous - progress has been achievable. As this progress continues, there is little doubt that more surprises remain in store.

Acknowledgments. I wish to thank Chuck Newman for a long and enjoyable collaboration on these questions, and for helpful comments on the manuscript. This work was supported in part by National Science Foundation Grant DMS01-02541.

\section{References}

1. V. Cannella and J.A. Mydosh: Phys. Rev. B 6, 4220 (1972).

2. K. Binder and A.P. Young: Rev. Mod. Phys. 58, 801 (1986).

3. S. Kirkpatrick, C.D. Gelatt, Jr., and M.P. Vecchi: Science 220, 671 (1983).

4. M. Mézard and G. Parisi: J. Phys. Lett. 46, L771 (1985).

5. Y. Fu and P.W. Anderson: J. Phys. A19, 1605 (1986).

6. M. Mézard and G. Parisi: J. Phys. 47, 1285 (1986).

7. J.J. Hopfield: Proc. Natl. Acad. Sci. USA 79, 2554 (1982).

8. D.J. Amit, H. Gutfreund, and H. Sompolinsky: Phys. Rev. Lett. 55, 1530 (1985).

9. P.W. Anderson: Proc. Natl. Acad. Sci. USA 80, 3368 (1983).

10. P.W. Anderson and D.L. Stein: 'Broken Symmetries, Dissipative Structures, Emergent Properties, and Life'. In: Self-Organizing Systems, ed. by F. Yates (Plenum, NY, 1985).

11. D.S. Rokhsar, D.L. Stein, and P.W. Anderson: J. Mol. Evol. 23, 119 (1986).

12. D.L. Stein: Proc. Natl. Acad. Sci. USA 82, 3670 (1985).

13. J.D. Bryngelson and P.G. Wolynes: Biopolymers 30, 177 (1990).

14. M. Mézard, G. Parisi, and M.A. Virasoro: Spin Glass Theory and Beyond (World Scientific, Singapore 1987).

15. D.L. Stein: Spin Glasses and Biology (World Scientific, Singapore 1992).

16. H. Nishimori: Statistical Physics of Spin Glasses and Information Processing (Oxford University Press, Oxford, 2001).

17. S.F. Edwards and P.W. Anderson: J. Phys. F 5, 965 (1975).

18. G. Toulouse: Commun. Phys. 2, 115 (1977).

19. P.W. Anderson: J. Less-Common Metals 62, 291 (1978).

20. D. Sherrington and S. Kirkpatrick: Phys. Rev. Lett. 35, 1792 (1975).

21. D.J. Thouless, P.W. Anderson, and R.G. Palmer: Phil. Mag. 35, 593 (1977). 
22. J.R.L. de Almeida and D.J. Thouless: J. Phys. A 11, 983 (1978).

23. H. Sompolinsky and A. Zippelius: Phys. Rev. Lett. 47, 359 (1981).

24. H. Sompolinsky, Phys. Rev. Lett. 47, 935 (1981).

25. H. Sompolinsky and A. Zippelius, Phys. Rev. B 25, 6860 (1982).

26. G. Parisi: Phys. Rev. Lett. 43, 1754 (1979).

27. G. Parisi: Phys. Rev. Lett. 50, 1946 (1983).

28. M. Mézard, G. Parisi, N. Sourlas, G. Toulouse, and M. Virasoro: Phys. Rev. Lett. 52, 1156 (1984).

29. M. Mézard, G. Parisi, N. Sourlas, G. Toulouse, and M. Virasoro: J. Phys. (Paris) 45, 843 (1984).

30. C.M. Newman and D.L. Stein: J. Stat. Phys. 106, 213 (2002).

31. C.M. Newman and D.L. Stein: "Topical Review: Ordering and Broken Symmetry in Short-Ranged Spin Glasses", submitted to Journal of Physics: Condensed Matter.

32. C.M. Newman and D.L. Stein: "Ultrademocracy of Ground States in the InfiniteRanged Spin Glass", submitted to Phys. Rev. Lett.

33. M.E. Fisher and R.R.P. Singh: 'Critical Points, Large-Dimensionality Expansions, and the Ising Spin Glass'. In: Disorder in Physical Systems, ed. by G. Grimmett and D.J.A. Welsh (Clarendon Press, Oxford, 1990) pp. 87-111.

34. M.J. Thill and H.J. Hilhorst: J. Phys. I 6, 67 (1996).

35. A.T. Ogielski: Phys. Rev. B 32, 7384 (1985).

36. A.T. Ogielski and I. Morgenstern: Phys. Rev. Lett. 54, 928 (1985).

37. N. Kawashima and A.P. Young: Phys. Rev. B 53, R484 (1996).

38. E. Marinari, G. Parisi, and F. Ritort: J. Phys. A 27, 2687 (1994).

39. W.L. McMillan: J. Phys. C 17, 3179 (1984).

40. A.J. Bray and M.A. Moore: Phys. Rev. B 31, 631 (1985).

41. A.J. Bray and M.A. Moore: Phys. Rev. Lett. 58, 57 (1987).

42. D.S. Fisher and D.A. Huse: Phys. Rev. Lett. 56, 1601 (1986).

43. D.A. Huse and D.S. Fisher: J. Phys. A 20, L997 (1987).

44. D.S. Fisher and D.A. Huse: J. Phys. A 20, L1005 (1987).

45. D.S. Fisher and D.A. Huse: Phys. Rev. B 38, 386 (1988).

46. A.A. Middleton: Phys. Rev. Lett. 83, 1672 (1999).

47. M. Palassini and A.P. Young: Phys. Rev. B 60, R9919 (1999).

48. A.K. Hartmann: Eur. Phys. J. B 13, 539 (2000).

49. C.M. Newman and D.L. Stein: Phys. Rev. Lett. 84, 3966 (2000).

50. C.M. Newman and D.L. Stein: Commun. Math. Phys. 224, 205 (2001).

51. M. Palassini and A.P. Young: Phys. Rev. Lett. 83, 5126 (1999).

52. E. Marinari and G. Parisi: Phys. Rev. B 62, 11677 (2000).

53. C.M. Newman and D.L. Stein: Phys. Rev. Lett. 76, 515 (1996).

54. C.M. Newman and D.L. Stein: Phys. Rev. Lett. 76, 4821 (1996).

55. C.M. Newman and D.L. Stein: 'Thermodynamic Chaos and the Structure of ShortRanged Spin Glasses'. In: Mathematics of Spin Glasses and Neural Networks, ed. by A. Bovier and P. Picco (Birkhäuser, Boston, 1997) pp. 243-287.

56. C.M. Newman and D.L. Stein: Phys. Rev. E 55, 5194 (1997).

57. C.M. Newman and D.L. Stein: Phys. Rev. E 57, 1356 (1998).

58. C.M. Newman and D.L. Stein: Phys. Rev. B 46, 973 (1992).

59. H. Simon: 'The Organization of Complex Systems'. In: Hierarchy Theory - The Challenge of Complex Systems, ed. by H.H. Pattee (George Braziller, NY, 1973).

60. M. Aizenman and J. Wehr: Commun. Math. Phys. 130, 489 (1990). 